\title{
Two-Channel Kondo Lattice: An Incoherent Metal
}

\author{
Mark Jarrell, ${ }^{1}$ Hanbin Pang, ${ }^{1}$ D. L. Cox, ${ }^{2,3}$ and K. H. Luk ${ }^{4}$ \\ ${ }^{1}$ Department of Physics, University of Cincinnati, Cincinnati, Ohio 45221 \\ ${ }^{2}$ Department of Physics, The Ohio State University, Columbus, Ohio 43202 \\ ${ }^{3}$ Institute for Theoretical Physics, University of California, Santa Barbara, California 93106-4030 \\ ${ }^{4}$ Research Centre, The Hong Kong University of Science and Technology, Clear Water Bay, Kowloon, Hong Kong
}

(Received 6 May 1996)

The paramagnetic phase of the two-channel Kondo lattice model is examined with a quantum Monte Carlo simulation in the limit of infinite dimensions. We find non-Fermi-liquid behavior at low temperatures including a finite low-temperature single-particle scattering rate, no Fermi distribution discontinuity, and zero Drude weight. However, the low-energy density of electronic states is finite. We label our model system in this phase an "incoherent metal." We discuss the relevance of our results for concentrated heavy fermion metals with non-Fermi-liquid behavior. [S0031-9007(96)00886-1]

PACS numbers: 75.30.Mb, 71.27.+a, 75.10.Dg

The Fermi liquid theory of Landau has provided a remarkably robust paradigm for describing the properties of interacting fermion systems such as liquid ${ }^{3} \mathrm{He}$ and alkali metals (e.g., sodium). The key notion of this theory is that the low lying excitations of the interacting system possess a 1:1 map to those of the noninteracting system and hence are called "quasiparticles." In the metallic context, one may think of the quasiparticles as propagating electronlike wave packets with renormalized magnetic moment and effective mass reflecting the "molecular field" of the surrounding medium. A sharp Fermi surface remains in the electron occupancy function $n_{\vec{k}}$ which measures the number of electrons with a given momentum, and for energies $\omega$ and temperatures $T$ asymptotically close to the Fermi surface the excitations have a decay rate going as $\omega^{2}+\pi^{2}\left(k_{B} T\right)^{2}$, which is much smaller than the quasiparticle energy, and generally translates into a $T^{2}$ contribution to the electrical resistivity $\rho(T)$. This theory has proven useful in describing phase transitions within the Fermi liquid, such as superconductivity which is viewed as a pairing of Landau quasiparticles in conventional metals such as aluminum.

The Fermi liquid paradigm appears now to be breaking down empirically in numerous materials, notably the quasi-two-dimensional cuprate superconductors [1] and a number of fully three-dimensional heavy Fermion alloys and compounds [2]. In these systems such anomalies as a conductivity with linear dependence on $\omega, T$ and logarithmically divergent linear specific heat coefficients are often observed. If the quasiparticle paradigm indeed breaks down, this may require completely new concepts to explain the superconducting phases of these materials. While the Luttinger liquid theory provides an elegant way to achieve non-Fermi-liquid theory in one dimension (with, e.g., no jump discontinuity in $n_{\vec{k}}$, and separation or unbinding of spin and charge quantum numbers), this results from the special point character of the Fermi surface. Whether the essential spin-charge separation may "bootstrap" into higher dimensions remains unclear [3].
Among the remaining theories to explain experiment are those based upon proximity to a zero-temperature quantum critical point [4], those based upon disorder induced distributions of Kondo scales in local moment systems [5], and those which hope to explain the physics from impurity to lattice crossover effects in the multichannel Kondo model [6]. Notably lacking for dimensions higher than one are rigorous solutions to microscopic models which display non-Fermi-liquid behavior [7].

In this Letter, we present the first rigorous solution of the two-channel Kondo lattice model in infinite spatial dimensions. We find that the paramagnetic phase of this model is an "incoherent metal" with finite density of states at the Fermi energy and finite residual resistivity. The excitation spectrum is non-Fermi-liquidlike; in particular, there is a finite lifetime for electrons at the Fermi energy, an ill defined quasiparticle mass, a linear low-temperature electrical resistivity with a finite residual value, and no discontinuity in $n_{\vec{k}}$. We find that physical quantities may be suitably scaled with a lattice Kondo scale $T_{0}$ that is significantly enhanced over the impurity limit. We discuss the possible relevance of these results to understand transport properties of concentrated heavy electron materials.

The two-channel Kondo impurity model consists of two identical species of noninteracting electrons antiferromagnetically coupled to a spin $1 / 2$ impurity. Non-Fermiliquid behavior results because of the inability to screen out the impurity spin: it is energetically favorable for both conduction electron bands to couple to the impurity which gives a spin $1 / 2$ complex on all length scales. As a result, the ground state is degenerate and the excitation spectrum non-Fermi-liquidlike. In contrast, the single-channel Kondo model has a singlet ground state with the impurity spin screened out, and a Fermi liquid excitation spectrum corresponding to the removal of one conduction state from the system. On extension to the lattice and ignoring the renormalization of the environment around each spin, the array of single-channel model singlets would simply renormalize the potential scattering. In contrast, the 
array of many body doublets in the two-channel case would give rise to a dynamical spin-disorder scattering in the absence of any cooperative transition that lent coherence to the spin array. We might thus anticipate a finite residual resistivity and other non-Fermi-liquid properties in the paramagnetic phase [8].

The Hamiltonian for the two-channel Kondo lattice is

$$
\begin{aligned}
H= & J \sum_{i, \alpha} \mathbf{S}_{i} \cdot \mathbf{s}_{i, \alpha}-\frac{t^{*}}{2 \sqrt{d}} \sum_{\langle i j\rangle, \alpha, \sigma}\left(c_{i, \alpha, \sigma}^{\dagger} c_{j, \alpha, \sigma}+\text { H.c. }\right) \\
& -\mu \sum_{i, \alpha, \sigma} c_{i, \alpha, \sigma}^{\dagger} c_{i, \alpha, \sigma}
\end{aligned}
$$

where $c_{i, \alpha, \sigma}^{\dagger}\left(c_{i, \alpha, \sigma}\right)$ creates (destroys) an electron on site $i$ in channel $\alpha=1,2$ of $\operatorname{spin} \sigma, \mathbf{S}_{i}$ is the Kondo spin on site $i$, and $\mathbf{s}_{i, \alpha}$ are the conduction electron spin operators for site $i$ and channel $\alpha$. The sites $i$ form an infinitedimensional hypercubic lattice. Hopping is limited to nearest neighbors with hopping integral $t \equiv t^{*} / 2 \sqrt{d}$; the scaled hopping integral $t^{*}$ determines the energy unit and is set equal to $1\left(t^{*}=1\right)$. Thus, on each site the Kondo spin mediates spin interaction between the two different channels. This problem is nontrivial, and, for the region of interest in which $J>0$ and $T \ll J, t^{*}$ it is describable only with nonperturbative approaches. Clearly some simplifying method which allows for a solution of the lattice problem in a nontrivial limit is necessary.

Such a method was proposed by Metzner and Vollhardt [9] who observed that the renormalizations due to local two-particle interactions become purely local as the coordination number of the lattice increases. More precisely, the irreducible single-particle self-energy $\Sigma_{\vec{k}}(z)$ and therefore the irreducible two-particle self-energy $\Gamma_{\vec{k}, \vec{k}+\vec{q}}\left(z, z^{\prime}\right)$ both become independent of momentum for large coordination number $(2 d \rightarrow \infty)[10,11]$. A further consequence of this is that the solution of most standard lattice models may be mapped onto the solution of a local correlated system coupled to an effective bath that is self-consistently determined [11-16]. We refer the reader to the above references and recent reviews for further details on the method [17].

In order to solve the remaining impurity problem, we use the Kondo impurity algorithm of Fye and Hirsch [18], modified to simulate the two-channel problem [19]. In the quantum Monte Carlo (QMC) approach the problem is cast into a discrete path formalism in imaginary time, $\tau_{l}$, where $\tau_{l}=l \Delta \tau, \Delta \tau=\beta / L$, and $L$ is the number of times slices. The values of $L$ used ranged from 8 to 96 , with the largest values of $L$ reserved for the largest values of $\beta$ since the time required by the algorithm increases like $L^{3}$. A sign problem was also encountered in the QMC process which limited our access to very low temperatures.

The Euclidean-time QMC results for the local greens function $G(\tau)$ were then analytically continued to real frequencies using the "annealing" maximum entropy method [20]. Here, we start from an exact result for the density of states (DOS) at high temperatures (i.e., a Gaussian) and use this as a default model to analytically continue the highest temperature data. The output is then used as a default model to analytically continue the data for the next lower temperature, and so on. As the temperature is lowered, only the low-energy features change, so very little additional information needs to be added at each temperature. To ensure that we have the best possible results from this procedure, we systematically improve the precision of the QMC data (by running longer) until the continued spectra stabilize. The single particle self-energy may then be obtained by inverting the relation $G(\omega)=-i \sqrt{\pi} w(\omega+\mu-\Sigma(\omega))$, where $w(z)$ is the complex Fadeev function.

Most of our simulations were limited to the model at half filling of the conduction bands $(N=1.0$ for $J=$ $0.75,0.625,0.5,0.4)$; however, we have also studied the system away from half filling $(N=0.75$ and 0.50$)$ for two values of $J(J=0.75$ and 0.625$)$. The qualitative features of the single-particle properties in the paramagnetic phase of the model do not depend strongly upon filling. Error bars are ill defined for individual points in analytically continued spectra [20], and are less than 3\% for the other results presented here.

As the temperature is lowered, the local susceptibilities show evidence of Kondo screening: the screened local Kondo moment $T \chi(T)$ falls like $T \ln (T)$, and the local Kondo spin-conduction spin correlation indicates an antiparallel alignment $\left\langle\mathbf{S}_{i} \cdot \mathbf{s}_{i, \alpha}\right\rangle<0$; whereas local spin-spin correlation function between the channels indicates a weak parallel alignment $\left\langle\mathbf{s}_{i, 2} \cdot \mathbf{s}_{i, 1}\right\rangle>0$. By comparing the susceptibility of the local moment to the impurity susceptibility, we are able to estimate the Kondo scale $T_{0}$ from the defining relation $T_{0} \chi\left(T_{0}\right)=0.3$. We find that $T_{0} \simeq 0.85 \mathrm{~J} \exp \left(-1.01 t^{*} / J\right)$. For comparison, in the impurity limit we would anticipate $T_{0}^{\text {imp }} \simeq \pi^{-1 / 2} J \exp \left(-\sqrt{\pi} t^{*} / J\right)$. Hence, $T_{0}$ is collectively enhanced in the lattice, as found in the one-channel Anderson lattice problem near half filling [21-23]; we discuss our enhancement further below.

When the temperature is lowered below $T_{0}$, we find non-Fermi-liquid behavior in the single-particle properties of the model. For example, in Fig. 1 the derivative of the particle distribution function $n\left(\epsilon_{k}\right)=T \sum_{n} G\left(\epsilon_{k}, i \omega_{n}\right)$ is plotted. $d n\left(\epsilon_{k}\right) / d \epsilon_{k}=-T \sum_{n} 1 /\left[i \omega_{n}-\epsilon_{k}+\mu-\right.$ $\left.\Sigma\left(i \omega_{n}\right)\right]^{2}$ is calculated by numerically evaluating the sum. This saturates to a finite width distribution, in contrast to a Fermi liquid where it would display a dominant delta function contribution as $T \rightarrow 0$, and a Luttinger liquid [3] or marginal Fermi liquid [24] where it would have a singular divergence. Clearly we see no such features in Fig. 1.

In Fig. 2 we show one electron properties of the model. Figure 2(a) displays the single particle DOS. This has a finite value as $\omega, T \rightarrow 0$, with a peak away from the Fermi energy. Note that suppression of $N(\omega=0)$ leads 


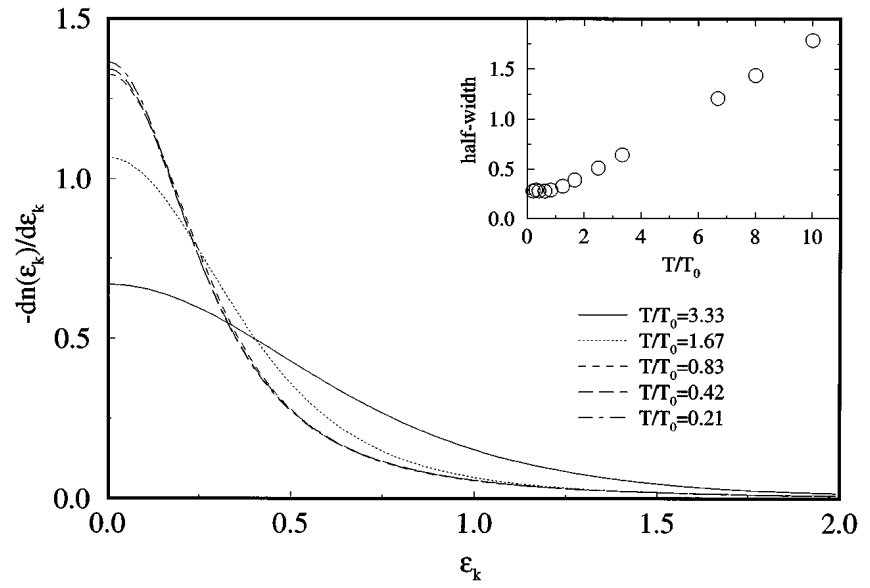

FIG. 1. Derivative of the particle distribution function $n\left(\epsilon_{k}\right)=T \sum_{n} G\left(\epsilon_{k}, i \omega_{n}\right)$ when $J=0.625$ and $N=1.0$. In a noninteracting system or one with a Fermi-liquid low temperature state, $-d n(0) / d \epsilon_{k} \propto Z^{2} \beta$ and the distribution should have a width $\propto T$ which is the width of the Fermi edge and is proportional to the scattering rate at the Fermi surface. However, in this case $-d n(0) / d \epsilon_{k}$ saturates at low $T$ and so does the width of $-d n\left(\epsilon_{k}\right) / d \epsilon_{k}$, shown in the inset, indicating that a Fermi liquid has not formed and that scattering rate remains finite when $\epsilon_{k}=\mu$ as $T \rightarrow 0$.

to an enhanced effective medium DOS which accounts qualitatively for our collectively enhanced $T_{0}$ values.

Novel behavior is seen in the real part of the one electron self-energy which has positive slope at $\omega \rightarrow 0$
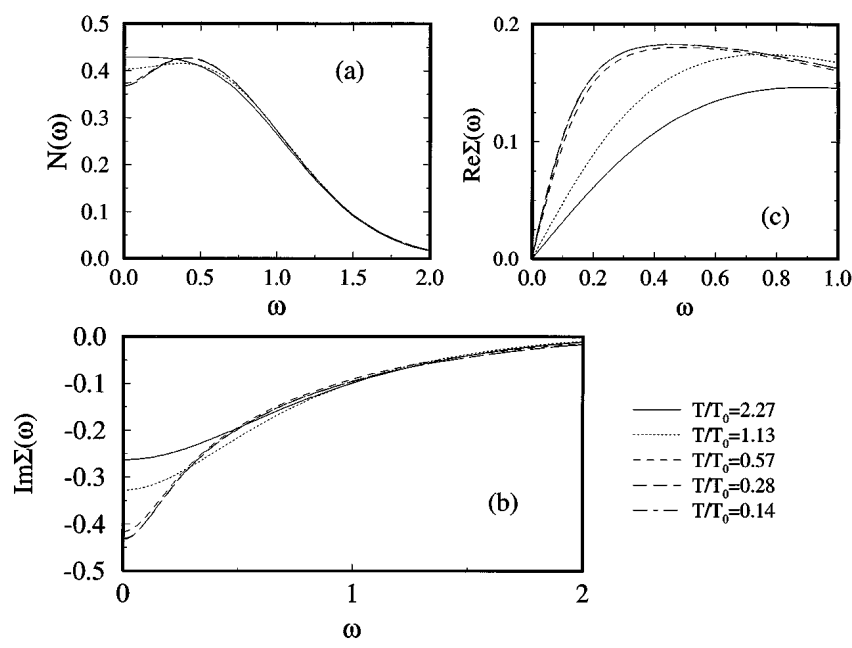

FIG. 2. Single-particle properties of the two-channel Kondo lattice in infinite dimensions when $J=0.750$ and $N=1.0$. (a) Single-particle DOS. At high temperatures $T \gg T_{0}$ (not shown), the DOS is a Gaussian, crossing over to the peaked distribution with relative suppression at $\omega \rightarrow 0$ for lower temperatures when $T \ll T_{0}$. (b) Imaginary part of the selfenergy. As the temperature is lowered the self-energy does not approach a Fermi-liquid form $\operatorname{Im} \Sigma(\omega) \propto-T^{2}-\omega^{2}$, but rather appears to approach the nonanalytic form (see text) $\operatorname{Im} \Sigma(\omega) \propto-c+(\omega)$. (c) The real part of the self-energy $\operatorname{Re} \Sigma(\omega)$ is also anomalous since its initial slope is positive indicating a quasiparticle renormalization factor which is greater than 1 .
[Fig. 2(c)]. For a Fermi liquid, this slope would be negative. The physical content is important: $Z=1 /(1-$ $\partial \operatorname{Re} \Sigma / \partial \omega)$ measures the overlap of the quasiparticle wave function with the original one-electron wave function having the same quantum labels. A positive slope leads to $Z>1$ or $Z<0$, indicating a breakdown of the quasiparticle concept. Concomitant with the finite width of $-d n / d \epsilon_{k}$ is a finite imaginary part to the low temperature self-energy [Fig. 2(b)]. This indicates that the oneelectron excitations are ill defined on approach to the Fermi surface, again ruling out a Fermi-liquid description. Since the low-temperature thermodynamic properties such as the specific heat, uniform magnetic susceptibility, and charge susceptibility display no evidence for a gap, we believe the observed behavior indicates a new kind of metallic state.

From our numerical results, one can show that the zero temperature self-energy must be nonanalytic. If the selfenergy is analytic everywhere on the real axis (and the upper half complex plane), then one may easily show that

$$
\lim _{T \rightarrow 0} \frac{\operatorname{Im} \Sigma\left(i \omega_{0}\right)}{\omega_{0}}=\left.\lim _{T \rightarrow 0} \frac{d \operatorname{Re} \Sigma(\omega)}{d \omega}\right|_{\omega=0},
$$

where $\omega_{0}=\pi T$ is the lowest fermionic Matsubara frequency along the imaginary axis. However, we find that when $T<T_{0}, \operatorname{Im} \Sigma\left(\omega_{0}\right) / \omega_{0}<0$ and is apparently divergent; whereas, from Fig. 2(c) it is apparent that $d \operatorname{Re} \Sigma(\omega) /\left.d \omega\right|_{\omega=0}>0$. Thus, $\Sigma(\omega)$ cannot be analytic at the origin of the complex plane. Given this, and the apparent shape of $\Sigma(\omega)$ in Fig. 2(b), we postulate the form $\operatorname{Im} \Sigma(\omega) \sim-c+|\omega|$.

The non-Fermi-liquid behavior also strongly effects experimentally relevant transport properties of the system. The electrical resistivity is shown in Fig. 3. We find that

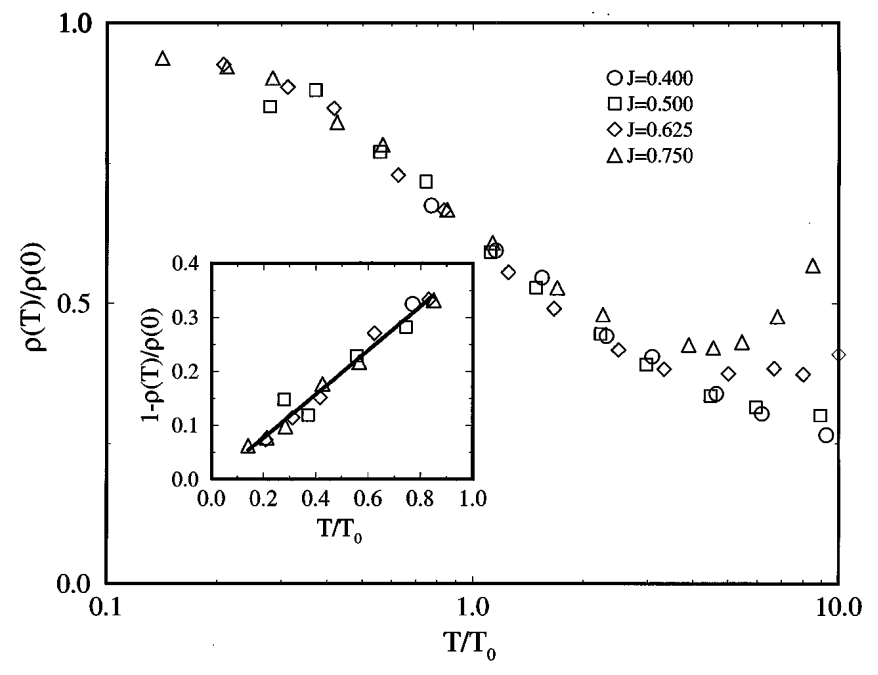

FIG. 3. Resistivity of the two-channel Kondo lattice. $\rho(T) / \rho(0)$ is plotted vs $T / T_{0}$ for various values of $J$. In the inset, the lowest temperature data (for $T / T_{0}<1$, shown as open circles) was fit by $\rho(T) / \rho(0)=1+B\left(T / T_{0}\right)^{\alpha}$, with $B=-0.4$ and $\alpha=1.03$. 
$\rho(T) / \rho(0)$ curves for different $J$ values collapse onto a universal scaling curve when plotted against $T / T_{0}$. As shown in the inset, $\rho(T) \simeq \rho(0)\left[1+B\left(T / T_{0}\right)\right]$ for $T \rightarrow$ 0 , with $B<0$ [25]. We interpret the finite value of $\rho(0)$ together with $-\operatorname{Im} \Sigma(0,0)$ as "spin-disorder scattering" off of the degenerate screening clouds centered about each local moment spin. Given the finite density of one particle excitations at the Fermi energy, this finite residual resistivity is indicative of an "incoherent metal phase" brought about by the disordered spin degrees of freedom, in qualitative agreement with results obtained with a Lorentzian bare conduction DOS (which does not self-consistently renormalize) [8]. We conjecture that the antiferromagnetism at half filling will lift the disorder at each site and produce an insulating state due to the cell doubling.

We have also computed the optical conductivity (not shown) and find that it displays vanishing Drude weight at low temperature together with a finite frequency peak $(\omega \sim 0.6 J)$. Both these features again support our interpretation in terms of a new kind of non-Fermi-liquid metallic state.

We find a commensurate antiferromagnetic transition at half filling for the simple nearest neighbor hopping model. We can suppress this phase by adding next neighbor hopping along each principal axis which will not alter our conclusions for the paramagnetic phase. The antiferromagnetic order is suppressed away from half filling. The complete phase diagram of the model will be explored in a separate publication.

Finally, we mention the possible applicability of our results to concentrated heavy fermion systems. Three systems display resistivity of the form $\rho(T) \approx \rho(0)[1+$ $\left.B\left(T / T_{0}\right)^{\alpha}\right]$ for $T<T_{0}$, with $\rho(0)$ of order the unitarity limit and $\alpha \approx 1$. They are $\mathrm{UCu}_{5-x} \mathrm{Pd}_{x}$ (with $B<0$ ) [2], $\mathrm{UBe}_{13}(B>0)[26,27,28]$, and $\mathrm{CeCu}_{2} \mathrm{Si}_{2}(B>$ 0) [29]. While $\mathrm{UCu}_{5-x} \mathrm{Pd}_{x}$ is a possible example of the distribution of Kondo scales scenario [5], $\mathrm{UBe}_{13}$ and $\mathrm{CeCu}_{2} \mathrm{Si}_{2}$ are ordered compounds which have been proposed as possible two-channel lattice systems (see Refs. [6b,c]; it is conceivable that $1 / d$ corrections can effect the sign change of $B$ relative to our results. Another key difference with our model is that we assume global $\left.\mathrm{SU}(2)\right|_{\text {spin }} \times\left.\mathrm{SU}(2)\right|_{\text {channel }}$ symmetry, while in the real materials these can only be local symmetries.

We acknowledge useful discussions with F. Anders, W. Chung, A. Georges, M. Ma, A. J. Millis, and W. Putikka. M.J. and H.P. acknowledge the support of NSF Grants No. DMR-9406678 and No. DMR-9357199. D. L. C. acknowledges the support of the U.S. Department of Energy, Division of Basic Energy Sciences, Office of Materials Research, and, at the ITP, by NSF Grant No. PHY94-07194. Computer support was provided by the Ohio Supercomputer Center.
[1] For a good overview, see High Temperature Superconductivity, edited by K. Bedell et al. (Addison-Wesley, Redwood City, CA, 1990).

[2] For a recent review, see M. B. Maple et al., J. Low Temp. Phys. 99, 223 (1995).

[3] P. W. Anderson and Y. Ren, in Ref. [1], p. 3.

[4] M. A. Continentino, Phys. Rev. B 47, 11587 (1993); A. J. Millis, Phys. Rev. B 48, 7183 (1993); A. M. Tsvelik and M. Reizer, Phys. Rev. B 48, 9887 (1993).

[5] V. Dobrosavljević, T. R. Kirkpatrick, and G. Kotliar, Phys. Rev. Lett. 69, 1113 (1992); O. O. Bernal et al., Phys. Rev. Lett. 75, 2023 (1995); E. Miranda, V. Dobrosavljević, and G. Kotliar (to be published).

[6] (a) P. Nozières and A. Blandin, J. Phys. (Paris) 41, 193 (1980); (b) D. L. Cox, Phys. Rev. Lett. 59, 1240 (1987); (c) Physica (Amsterdam) 186B-188B, 312 (1993).

[7] Q. Si and G. Kotliar, Phys. Rev. Lett. 70, 3143 (1993); Phys. Rev. B 48, 13881 (1993); Q. Si, et al., Phys. Rev. Lett. 72, 2761 (1994).

[8] D. L. Cox, Physica (Amsterdam) (to be published).

[9] W. Metzner and D. Vollhardt, Phys. Rev. Lett. 62, 324 (1989).

[10] E. Müller-Hartmann, Z. Phys. B 74, 507 (1989).

[11] U. Brandt and C. Mielsch, Z. Phys. B 75, 365 (1989); 79, 295 (1990); 82, 37 (1991).

[12] V. Janiš, Z. Phys. B 83, 227 (1991).

[13] C. Kim, Y. Kuramoto, and T. Kasuya, J. Phys. Soc. Jpn. 59, 2414 (1990).

[14] V. Janiš and D. Vollhardt, Int. J. Mod. Phys. B 6, 713 (1992).

[15] M. Jarrell, Phys. Rev. Lett. 69, 168 (1992).

[16] A. Georges and G. Kotliar, Phys. Rev. B 45, 6479 (1992).

[17] Th. Pruschke, M. Jarrell, and J. K. Freericks, Adv. Phys. 42, 187 (1995); A. Georges, G. Kotliar, W. Krauth, and M. Rozenberg, Rev. Mod. Phys. 68, 13 (1996).

[18] R. M. Fye and J. E. Hirsch, Phys. Rev. B 38, 433 (1988).

[19] K.-H. Luk, Mark Jarrell, and D. L. Cox, Phys. Rev. B 50, 15864 (1994).

[20] For a review of the maximum entropy method of analytic continuation, see M. Jarrell and J.E. Gubernatis, Phys. Rep. 269, 133 (1996).

[21] T. M. Rice and K. Ueda, Phys. Rev. B 34, 6420 (1986).

[22] N. Grewe, Th. Pruschke, and H. Keiter, Z. Phys. B 71, 75 (1988).

[23] M. Jarrell, Phys. Rev. B 51, 7429 (1995).

[24] C. M. Varma, P. B. Littlewood, S. Schmitt-Rink, E. Abrahams, and A.E. Ruckenstein, Phys. Rev. Lett. 63, 1996 (1989).

[25] We cannot rule out the appearance of a different power law for $T / T_{0} \leq 0.14$.

[26] See, for example, Fig. 25 and the discussion below in H. R. Ott, Progress in Low Temperature Physics, edited by D.F. Brewer (Elsevier, Amsterdam, 1987), Vol. XI, p. 216.

[27] M. C. Aronson, J. D. Thompson, J. L. Smith, Z. Fisk, and M. W. McElfresh, Phys. Rev. Lett. 63, 2311 (1989).

[28] F. Steglich (private communication).

[29] F. Steglich, et al., Physica (Amsterdam) (to be published). 